\title{
Reliability Analysis of ABB IRB 4600-40-255 Polishing Robot with Six Degree of Freedom
}

\author{
Jing-jing Zhao, You-qiang Wang
}

School of Mechanical Engineering, Qingdao University of Technology,Qingdao 266520 China

\begin{abstract}
Keywords: Polishing Robot with Six Degree of Freedom,Failure,Reliability Analysis,Fault Tree Analysis.

Abstract: Fault tree analysis,as an effective method for reliability analysis and evaluation of engineering systems,provided an effective method for failure analysis of polishing robots with six degree of freedom. Through the qualitative and quantitative analysis of the fault tree, it is proposed that the damage of the control electrical system is the main reason for the failure of polishing robots with six degree of freedom. This article aims to make a more comprehensive and systematic analysis on the reliability of the polishing robots with six degree of freedom, which has an important reference value for the design of the polishing robot.
\end{abstract}

\section{Introduction}

Polishing robots with six degree of freedom are mainly used in grinding,assembly,painting, handling and other occasions, which can effectively improve productivity and ensure production's quality and safety,occupying a large share of the industrial robot market.The four major families of industrial robots (ABB,KUKA, FANUC,YASKAWA) are concentrated in the Europe, which have a monopoly on the core components. In this paper,ABB IRB 4600-40-255 robots belonging to the joint robot, in which the maximum handling weight is $40 \mathrm{~kg}$ and the maximum working radius is $2.55 \mathrm{~m}$. Polishing robot's joints are all rotating joints, which is the most common industrial robot configuration. Figure. 1 is a structure diagram of six-joint grinding industrial robot.

Polishing robot with six degree of freedom is a complex system including mechanical, electronic, electrical,hydraulic and pneumatic,computer and other types of components and control software.The reliability of components is to restrict its application in the field of high precision.At present,the design of industrial robots often only consider the performance index, while ignoring the reliability review,resulting frequent failures in industrial robots,so the study of reliability is more complicated. Most of the research directions are structural and control design, while the most important reliability of robots is rarely studied.Because once the robot fails to work, it may cause injury to the user.Therefore, it is of great practical significance to study the reliability of polishing robot with six degree of freedom [1].

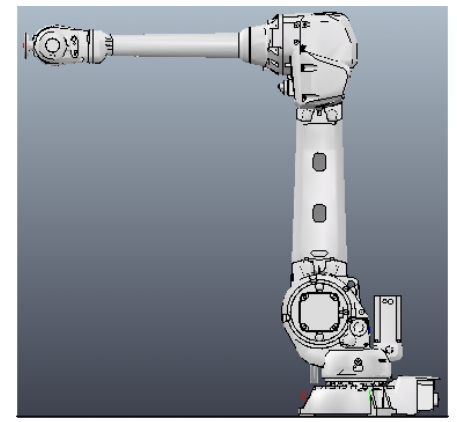

Figure 1. Structure diagram of six-joint grinding industrial robot

\section{Establish a reliability model}

The institutions shall complete the required mechanical action (movement) in a precise, timely and coordinated manner within the prescribed conditions and period.This ability is measured by 
probability as reliability. When studying the reliability of polishing robot with six degree of freedom, the correct reliability model should be established.If the robot is regarded as a system composed of several subsystems and components, the reliability of the whole system is expressed between them and the correlation degree is very close. The mathematical model expression is [3]:

$R(t)=f\left[R_{1}(t) R_{2}(t) \cdots R_{\mathrm{n}}(t)\right]$

Where, $R(t)$ is the system reliability; $R_{1}(t)$ is the component reliability function; and $\mathrm{N}$ is the number of components or components.

During the reliability prediction, some hypothetical methods can be used to estimate the reliability of the system and then to predict the reliability of the system. The reliability model of industrial robots is the concatenation of the reliability models of subsystems, while the subsystems are the concatenation of each component or the smallest part. The reliability indexes assigned to each subsystems have the following relations with the reliability indexes of the whole system [4]:

$f\left(R_{1} R_{2} \cdots R_{\mathrm{n}}\right) \cdots>R$

Where, $R_{1}$ is the reliability index assigned to the first subsystem.

Without any constraint,there will be innumerable solutions to the above formula.Considering the comprehensive system condition,economy and technology, we should restrain the above formula and get the solution.

Establishing a kinematic model is the basis for the exercise reliability analysis. First need to number the connecting rods and joints:the ground-mounted robot base number is 0 ,it's attached waist is numbered 1,then the small arms, big arms and wrist are numbered $2,3 \cdots \mathrm{n}$. In this paper, the connecting rod 6 is fixedly connected with the end effector. Figure. 2 is the coordinate system of the Polishing robot with six degree of freedom [5].

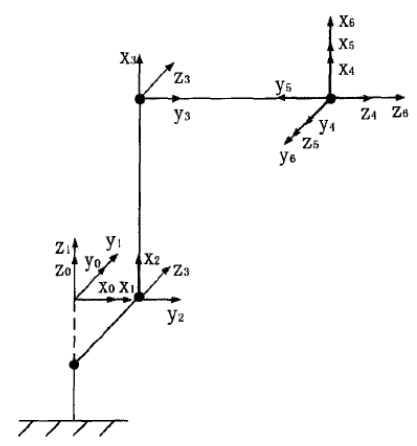

Figure 2. Six-joint grinding robot coordinate system

In most cases, the robot base needs to be fixed with the ground and has no relative displacement. Therefore, in order to analyze and calculate conveniently, the origin of the absolute coordinate system and the base coordinate system should be coincident, the direction is the same.

\section{V-rep Software Description}

Based on V-rep (Virtual Robot Experimentation Platform) simulation software analysis platform, the research content of this paper is the motion reliability analysis of polishing robot with six degree of freedom.V-rep is a very good open source robot simulation software,with a very powerful kinematics and dynamic analysis function, you can establish your own experimental environment and objects in the software and verify your algorithm. Supporting for external applications such as $\mathrm{C} / \mathrm{C}++$, Matlab, Python and other control applications, V-rep widely used in robotics, aerospace, automotive engineering, railway vehicles and equipment and construction machinery and other fields.

The ABB IRB 4600-40-255 six-joint polishing robot consists of six connecting rods and six rotating joints with six degrees of freedom. According to the range of each joint and the length of each link to get robot's working range as shown in Figure 3 and Figure 4, where $A=2872 \mathrm{~mm}, \mathrm{~B}=1735 \mathrm{~mm}$, $\mathrm{C}=1393 \mathrm{~mm}, \mathrm{D}=680 \mathrm{~mm}, \mathrm{E}=2202 \mathrm{~mm}, \mathrm{~F}=2552 \mathrm{~mm}$. 


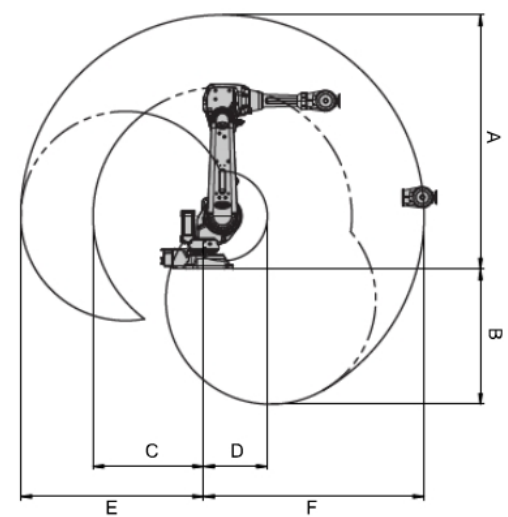

Figure 3. Working trajectory diagram of six-joint grinding robot

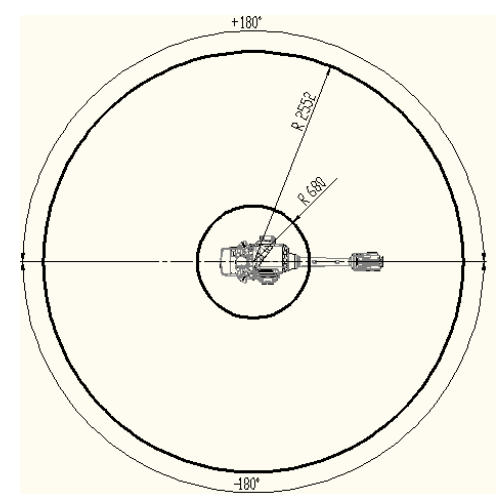

Figure 4. Top view of working trajectory of six-joint grinding robot

\section{Fault tree analysis}

Fault tree analysis is a common and practical method of system reliability analysis. The method is concise, intuitive and flexible and suitable for the failure analysis of polishing robot with six degree of freedom. Fault tree analysis takes the most undesirable failure state in the system as the top event, then finds out all possible causes (intermediate events) that lead to this fault state and then identifies all the possible causes that cause these intermediate failure events. And so on,until you find all the reasons that caused the part to fail. When the size of the fault tree is very large, it is more effective to solve it by the simulation method. Through the simulation, the importance of important components can be obtained. Since the six drive chains of the six-joint grinding robot play a decisive role in the failure of the end movement, so the entire system belongs to a series system.

The top event selected here is the motion failure of the polishing robot with six degree of freedom. The failure of the motion refers to the fact that the robot's end did not complete the predetermined position and posture and the corresponding velocity and acceleration. Fault tree analysis method has an important guiding role to find the weak links in the system and optimize the mechanical structure.The reliability of the robot system is analyzed from the aspects of subsystems and fault responsibility.Then, taking the control system with the most fault data as example,the fault tree analysis method is used to analyze the reliability of the control system. The most fundamental cause of the fault is found by layer by layer and some improvement measures are put forward according to the fault reason.

\section{Minimal cut set}

Using Semanderes algorithm,which is characterized by bottom-up,it is also known as "ascending". Each step is to use the set of computing rules to simplify, calculating the minimum is the minimum cut set. Minimal cut sets directly affect the reliability of the system,that is the weak links of the system. In order to improve the reliability and service life of the polishing robot, we should start with the minimum cut set. Obtained the logical relationship between the whole failure and failure of parts and 
the failure of parts and the failure of each part to establish the fault tree of the polishing robot with six degree of freedom. The minimal cut set that leads to the occurrence of a failure event is analyzed and obtains a model of reliability analysis in terms of motion reliability.

\section{Qualitative and quantitative reliability analysis}

Reliability analysis methods include qualitative analysis and quantitative analysis. Qualitative analysis generally finds out the causes of the fault, provided advice for the design and maintenance. Fault tree analysis focuses on qualitative analysis, but also can be quantitative analysis. Quantitative analysis is generally based on the application of mathematical statistics and other probabilistic methods. The generalized force and the generalized intensity of the interference model as the basic model to get the reliability of the product.The method of partial counting is used to analyze the repeatability and error of robot motion, which provides an important reference for error compensation and maintenance.

The purpose of the qualitative and quantitative analysis of the fault tree is to find all its smallest cut sets and to determine the probability of occurrence of the top and bottom events. Generally assume that the bottom events are independent of each other. The bottom and top events only consider two states, that is normal or fault. A minimum cut set of the fault tree represents a failure mode of the system.As long as there is a minimum cut set, the system is in a fault state. Assuming the system has a $K$ minimum cut set $K=\left(K_{1}, K_{2}, \cdots, K_{\mathrm{n}}\right)$. In the $K$ minimum cut set, the top event occurs whenever a minimum cut is taken.

\section{Main influencing factors and improvement measures}

In order to facilitate the reliability analysis, this paper divides the ABB IRB 4600-40-255 polishing robot into several subsystems, which respectively correspond to the different functions of each subsystem. Mechanical body system mainly includes the base of the robot, the balance cylinder, the big arm,the small arm,the wrist joint and other key parts and other mechanical parts and components, the driving system, the control electric system, the robot protection and the basic parts, the end grinding system, the pneumatic system and the lubrication system.

The breakdown frequency of each subsystem can be obtained and the fault frequencies of the control system are far more than other subsystems, which account for more than half of the total number of failures.Mainly because of the six-joint grinding robot control of the electrical system is more complex, including software and hardware systems. Most of the faults are caused by the poor quality of the grinding workpiece and the failure of the program upgrade.Therefore,the key to improve the reliability of the electrical system is to improve hardware equipment processing quality and improved procedure[5].Specific measures can be to improve the staff's skills, improve processing technology,increase quality inspection processes and so on.In the process of programming to fully understand the work of the robot can not omit every unexpected situation. In short, the most effective way to improve the reliability of the control of electrical system is to improve the circuit board and interface processing quality and improve the program.

The fault frequency of mechanical body system is also high,which mainly appears in the installation design of transmission gears. If the fault occurred will cause the robot joint suddenly out of control,so it is necessary to the pay attention to the mechanical body system. Drive system fault frequency is relatively high, the main fault is the motor or drive connection problems. Once the drive system fails, it will cause the robot downtime. In addition the lubrication system, robot protection and the basic parts will also have some failures. It's proportion is not very high and it's damage is generally less harmful. Of course, these reasons can not be overlooked.

Based on the above analysis, no matter which subsystem fails will affect the normal operation of polishing robot system. The damage caused by different faults may vary greatly. In case of failure all need to stop maintenance, which will affect the normal production. Therefore, no matter what the magnitude of the fault, it should be avoided as much as possible. 


\section{Conclusion}

(1) The fault tree analysis method is concise, intuitive and efficient. It is an effective method to analyze the failure of the polishing robot with six degree of freedom.

(2) The establishment of polishing robot failure fault tree considered a number of basic events. Through the analysis of the fault tree, we found the weak link of the polishing robot and concluded that the damage of control electrical system was the most major factor.

(3) The main factors affecting the failure of the polishing robot were determined by qualitative analysis and the corresponding improvement measures were put forward, which provided important help for preventing or reducing the failure of six-joint grinding robot. In industrial robots debugging or using stage, fault tree analysis can help maintenance personnel to diagnose faults, find out the causes of faults faster and improve maintenance schemes [8-9].

\section{Acknowledgement}

This research was financially supported by the National Science Foundation.

\section{References}

[1]Zhou Jian,Yang Liu,Zhang Zhijia.Reliability Analysis of industrial robots [J].Design and research, 2015(1):25-26.

[2] Huang Xingbao.Failure tree analysis of diaphragm coupling[J].Mechanical Drive,2014 (12):9698.

[3] Wang Gaiyun,Liang Fen,Zhu Mingri.Reliability analysis of welding robot based on fault tree[J]. 2008(24):272-274.

[4] Feng Xunping,Deng Zongquan.Fuzzy evaluation method for reliability analysis of pipeline robot [J]. Mechanical Design,2005(10):11-13.

[5] YangXiaolei.Reliability analysis and kinematic calibration of SR165-type industrial robot[D].20 15(6).

[6] Jiang Yi.Reliability analysis of 6R industrial robot based on virtual prototyping technology[D]. 2017(6).

[7] ChenWei.Reliability analysis and rigid-flexible coupling dynamic characteristics of truss robot [D].2017(4).

[8]Zhang Xiaojin,Chen Dong,Xie Liyang.Accuracy reliability analysis of six-DOF arc welding robot with Humontcallo method[J].2011:537-541.

[9] Shi Wei,Li Huaizheng,Chen Qiyu.Reliability analysis of industrial robot system based on Monte Carlo and Fault tree[J].2017(46):48-52. 